\title{
The Influence of Schematic Knowledge Differences on Memory Recall: How Do Different Cultural Backgrounds Impact Memory Recognition
}

\author{
Junchi Huang ${ }^{1, \dagger}$, Ziyu Wan ${ }^{2, *}, \dagger$, Jun Wang ${ }^{3, \dagger}$, Lan $\mathrm{Xu}^{4, \dagger}$ \\ ${ }^{1}$ University of California Santa Barbara, Santa Barbara, United States \\ ${ }^{2}$ University of California San Diego San Diego, United States \\ ${ }^{3}$ University of California San Diego, San Diego, United States \\ ${ }^{4}$ University of Melbourne, Melbourne, Australia \\ *Corresponding author. Email: ziwan@ucsd.edu \\ These authors contributed equally.
}

\begin{abstract}
Throughout years of studying the schematic knowledge influence or schema effect, we have a general understanding of how it manipulates our memory and causes some sorts of different levels of memory distortions. This study examines how the differences in schematic knowledge are going to influence people's memory. The present study involved 20 participants from 18 to 30 years old who had at least finished high school education. Participants in this experiment will either be domestic Chinese Students who have never studied abroad or studied in Western countries for less than a year, or international Chinese students who have studied one or more years in Western countries. Participants will be shown to two different backgrounds of dining halls either Chinese or Western dining halls and followed with a menu list including 10 dishes, and they will be tested on a memory recognition test of those dishes. In this case of study, both domestic and international Chinese students should have more background information with their corresponding dining halls and menus so that domestic Chinese are more familiar with Chinese dining hall and menu, while international students are more familiar with Western dining halls and menus, in which they should react faster and have a better accuracy rate when the test is corresponding to their schemata.
\end{abstract}

Keywords: Schematic information, Cultural background, Memory Recognition.

\section{INTRODUCTION}

Schematic knowledge is used often in our daily life from comprehending things to remembering events. Though schemata are not always perfect, they interfere with our central memory encoding processes and thus cause somewhat memory distortions [1]. Alba and Hasher evaluated how the schematic information influenced four of the memory encoding processes: selection, abstraction, interpretation, and integration. And all four encoding processes show that prior knowledge (schema) played a huge role in forming our memory or retrieving information from our memory. In addition, schematic knowledge is not something that we are born inert. Rather, it is a build-up information that we developed and establish through our experiences in life, and thus sometimes it may change and deviates as time goes [2].
The experiment led by Rubinova, Blank, Koppel, and Ost focused on finding how our established schema for unfamiliar events changed through exposure to different variants that they designed 4 different versions of stories and varied the orders and contents of them and tested the participants in 4 different time spans. In the experiment, the stories were based on a simplified and shortened passage of a Korean Wedding Ceremony. In brief, participants showed a gradual increase of accuracy of recall as the examples of stories go, which demonstrated that there was a build-up schema formation of the unfamiliar stories and helped us consolidate our memory formation as well. Besides, there were few memory errors of deviation from the original story shown than when it was tested with familiar stories [2]. In this case, it suggested that the build-up of new schema took over more of the attention when remembering events. 
Further research has done not only schematic knowledge's influence on encoding processes or developing new schema, but also its influence on people's everyday source monitoring tasks, the judgments of where the origin of the information is [3]. In this experiment, ninety-six pairs of sentences were selected from 327 sentences that one of the pairs will be used as the distractor, which they would not be presented in the actual study. Ninety-six of the sentences were evenly distributed into three categories of the sentence type (e.g., expected-lawyer statement, expected-doctor statement, and equally expected statement) and assigned into one of the sources that are either doctor or lawyer. Participants were shown to all 96 sentences one by one, that each followed with a source of the statement on a computer screen, and they will be tested on which sources the sentences belong to. In conclusion, the predicted interaction between the expectancy and sources were proved that the correct identification of sources was significantly higher when expectancy and its source matched, which demonstrated that schematic knowledge positively influences our memory recall on source monitoring. However, similar to the last experiment, Bayen and Spaniol did another experiment found a rather different result that though the schematic knowledge affects the source monitoring, its effect is relatively late in processing and schematic knowledge may largely depended on the person itself that the interpersonal differences can play a huge role [4]. Also, other research indicates that with the help of judgement of learning, the error of memory distortions may decrease significantly that caused by schematic information [5].

In addition, schematic information also has a profound influence on spatial memory found by Brewer and Treyens that when objects' s expectancy and saliency are high with its corresponding locations, the frequency of recall of both the objects and locations will be high as well [6]. Another experiment related to spatial memory and schemata was conducted by Hess and Slaughter in 1990. They conducted an experiment that studied how age played a role in spatial memory and their use of schema knowledge that young adults and old people were selected in this experiment. Organized and Unorganized kitchen scenes were shown to the participants and asked to take both the object recognition test and placement test (place the object to where it belongs in the scene). To conclude, when scene organization was low, subjects tended to place objects in a manner that is more consistent with their knowledge of the world, which proved that the schematic knowledge is tightly related to our spatial memory. And they also found that elimination of a meaningful spatial organization had a greater negative impact on the placement of high-likelihood objects in the older adults, but younger adults were better able to use the organization to enhance memory for low-likelihood objects. In this case, it indicates that older adults are more reliant on schematic information and more likely to use them than younger people [7].

With the knowledge of how schematic information can either enhance the possibility of retrieving information or cause memory errors, we are planning to dig deeper into the topic that whether cultural variations can also influence people's formation of schemata and memory retrieval, and how much of the schemata is culturally specific. In Carrell's review, it appears that there isn't enough evidence of generalizable content schemata that is culturally specific due to the existence of different cultural group may developed same schemata for certain things [8]. However, Qi and Roberts found the cultural differences when doing memory recognition test that comparing 10-year-old Chinese and Euro-Canadian children's memory recall of stories [9]. Both the Chinese and Euro-Canadian children watched a story involving both social- and individual-focused scenarios. Participants then did recall tests both immediately and delayed. The delayed experiment was tested five to seven days after the immediate test to eliminate the recency effect. As of result, Chinese children are better at remembering socially related events in the story than individuals. On the other hand, the Euro-Canadian children more accurately recall the individual related events of the story. In conclusion, in both the immediate and delayed cases, the cultural differences do play a role in what children are more likely to remember though in countries differences in delayed recall test isn't as significant.

Collectively, the past research on schematic information reviews schematic knowledge's influence on our encoding and retrieving of the memory. Understanding the effects of schematic knowledge helps us to establish the connection between memory recall and the difference of schemata. In our present study, we aim to keep investigating how cultural level schematic differences influence our memory recall like what Qi and Roberts did with Chinese and Euro-Canadian children. Therefore, our experiment is also mainly focusing on the aspect of schematic knowledge differences and memory recognition. In our study, two culturally different participant groups (domestic and international Chinese students) will be tested on recalling menu lists from either Chinese dining hall scene background or Western dining hall scene background, followed by a menu that contains both Chinese and English dishes with the same ratio. Experiments will then collect and measure the accuracy and the reaction time of each participant when they recall the dish from both backgrounds. We hypothesize that participants should be more familiar with their own culturally familiar backgrounds and menus, and have higher accuracy and faster recall when doing the test. That is to say, domestic Chinese students should react 
faster and have a better recall when the object is in a Chinese dining hall. Conversely, international students should react faster and have a better recall when the object is in the Western dining hall.

\section{METHODS}

\subsection{Participants}

The participants were 20 adults aged between 18 to 30 . There were 8 men $(40 \%)$ and 12 women $(60 \%)$ with a mean age of $21(\mathrm{SD}=1.747)$. Ten participants $(50 \%)$ did not have any previous study abroad experience. Ten participants $(50 \%)$ had study abroad experience (equal to or larger than 1 year). All participants were invited to participate in this experiment by friends or classmates with no reward. All participants consented to the participation and the study was approved by the local ethics committee.

\subsection{Materials}

Materials for the experiment are on-screen only, including a PsychoPy program and an online questionnaire used to collect participants' demographic information.

In this experiment, PsychoPy was used to create the platform (a program) where participants could perform. In the PsychoPy program, two dining hall pictures and two menus were shown. The dining hall pictures were a picture of a Chinese-style dining hall and a picture of a Western-style dining hall. In each menu, 5 Chinese traditional dishes (e.g., "Xiaolongbao") and 5 Western traditional dishes (e.g., "Cheeseburger") were presented both in English and in Chinese. All the dishes were randomly chosen from a list of 20 Chinese traditional dishes and a list of 20 Western traditional dishes. The order of 10 dishes on the menu was also randomized.

The PsychoPy program also contains two recognition tests. In each test, 12 dishes showed up on the screen with a question asking about the familiarity ("Have you seen this word before?") one by one. The dishes that showed up in the recognition test were randomly chosen from 2 lists. One list contained the dishes that were in the previous menu; the other contains dishes that were not in the previous menu. The ratio between the numbers of dished chosen from two lists was 50:50. The online questionnaire contains 3 demographic questions - participant's gender, age, and the number of years of living abroad.

\subsection{Procedure}

The experiment took place both online and in person. Some participants finished the experiment by downloading the PsychoPy program and perform it on their laptop or PC. Then, they sent the data to the experimenters for further analysis. Some other participants finished the experiment by performing it on the experimenters' computer. No matter which kind of experiment form (online or in-person) participants chose to use, when they got access to the PsychoPy program, they started with entering Participant ID (made up by themselves or the experimenters) and their session. After signing the consent form to agree to participate in the experiment, participants read the instruction of the experiment in English. The formal experiment started.

In the experiment, participants first saw the picture of the Chinese dining hall $(\mathrm{CDH})$ and a sentence indicating that this picture showed a Chinese dining hall. Then, they saw a menu with 10 dishes. Among them, 5 were Chinese traditional dishes, and 5 were Western traditional dishes. After 5 seconds, the menu disappeared, and the recognition test started. Participants were given a dish name and asked to evaluate whether they saw the dish in the menu they had just seen. Then, they rated the confidence level (5 levels in total) of their answer. This recognition process repeated for 12 dishes. After completing the recognition test, the second trial started. Participants were given a picture of the Western dining hall (WDH) and a sentence indicating that this picture showed a western dining hall. Similar to the first trial, after seeing the picture for 5 seconds, participants started the recognition test.

When participants completed 2 sessions, the PsychoPy program was over. They sent the data collected by PsychoPy to the experimenter. The experimenter gave them a $\mathrm{QR}$ code, which linked to an online questionnaire. In the questionnaire, participants were required to provide their age, gender, and the number of years they stayed abroad. They also provided their Participant ID in the questionnaire. After filling the form, the debriefing process started. Participants were given the goal and theory of the experiment by the experimenter. Participants were thanked and dismissed after the debriefing section.

\subsection{Data Analysis}

The threshold for statistical significance was set at $\mathrm{p}$ $<0.05$ for all analyses. Participant characteristics were analyzed using descriptive statistics. A 2 x 2 repeatedmeasures ANOVA was used to measure the effect of study abroad experience on recalling dish names from the menu, based on different dining hall pictures. The dependent variable is the accuracy for the recognition test and the response time for each selection in the recognition test. A post hoc test was also used to see the detailed effect of individual independent variables and combined. 


\section{RESULTS}

The mean accuracy and RTs of Chinese domestic and international students in the Chinese and Western dining hall backgrounds are illustrated in Figure 1 and Figure 2, respectively. The means and standard deviations of accuracy and RTs for students are shown in Table 1.

A repeated-measures ANOVA was conducted to compare the accuracy between two groups of students in Chinese and Western dining hall conditions. There was a significant difference of accuracy within the interaction of study abroad experiences and dining hall backgrounds, $F(1,9)=10.00, p=.01, \eta^{2}=.12$. However, there was no significant difference of accuracy in study abroad experiences $(F(1,9)=2.65$, p $\left.=.14, \eta^{2}=.12\right)$ and dining hall backgrounds $(\mathrm{F}(1,9)=$ $\left.0.38, \mathrm{p}=.55, \eta^{2}=.01\right)$. Following Cohen's criteria (1988), these constitute a medium to large effect for the interaction of study abroad experiences and dining hall backgrounds. It suggested that the interaction has a significant impact on the mean accuracy of the tests.

Post-hoc tests with Bonferroni corrections for multiple accuracy comparisons were used to analyze further the effects of interactional contexts on study experiences and dining hall backgrounds. It demonstrates that in $\mathrm{CDH}$ conditions, the difference of accuracy between domestic and international students was broadly significant, $\mathrm{p}=.07$. However, there were no other significant results.

Another repeated-measures ANOVA was conducted to compare the RTs between the same conditions as before. There was a significant difference of RTs in dining hall conditions, $F(1,9)=10.00, p<.01, \eta^{2}$ $=.163$. Nevertheless, there was no significant difference in univariate conditions of study experiences $(\mathrm{F}(1,9)=$ 2.53, $\left.\mathrm{p}=.15, \eta^{2}=.10\right)$ and interaction of study experiences and dining hall backgrounds $(F(1,9)=2.86$, $\left.\mathrm{p}=.13, \eta^{2}=.06\right)$. According to Cohen's criteria (1988), these suggest a significant large effect of dining hall backgrounds on the mean RTs.

The effects of multiple comparisons of RTs for dining hall backgrounds were further analyzed by posthoc tests with Bonferroni corrections. It illustrates that the RTs in $\mathrm{CDH}$ were significantly larger than in $\mathrm{WDH}$, $\mathrm{p}<.01$.

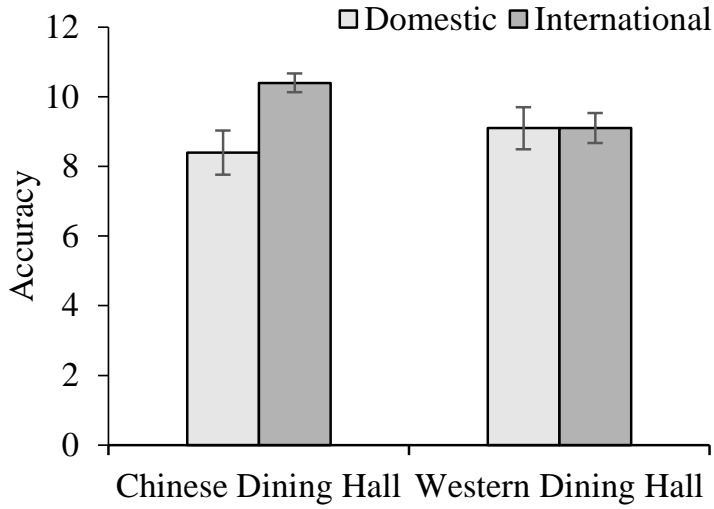

Figure 1. Mean Accuracy of Domestic and International Students in Chinese and Western Dining Hall Backgrounds

Note. Chinese Dining Hall $=$ Chinese dining hall background; Western Dining Hall = Western dining hall background; Accuracy = accuracy of memory test. Error bars represent standard errors.

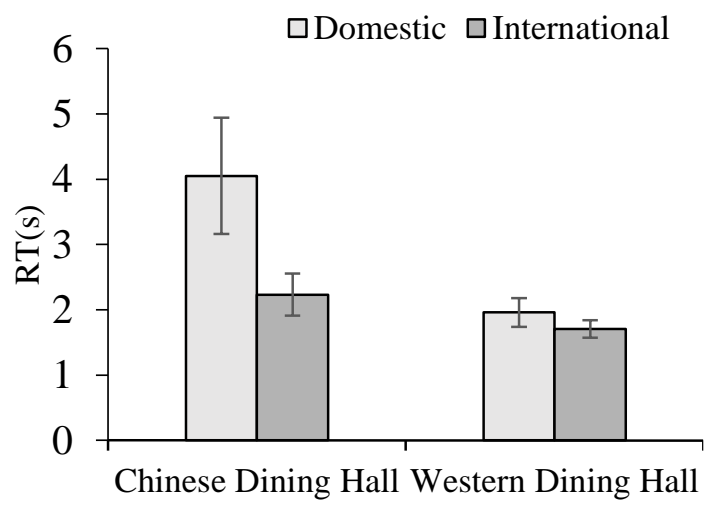

Figure 2. Mean Reaction Times of Domestic and International Students in Chinese and Western Dining Hall Backgrounds

Note. Chinese Dining Hall $=$ Chinese dining hall background; Western Dining Hall = Western dining hall background; RT = Reaction time. Error bars represent standard errors.

Table 1. Mean Accuracy and Reaction Times of

Domestic and International Students in Chinese and Western Dining Hall Backgrounds

\begin{tabular}{lllll}
\hline & \multicolumn{2}{c}{ Domestic } & \multicolumn{2}{l}{ International } \\
\cline { 2 - 5 } & $M$ & $S D$ & $M$ & $S D$ \\
\hline CDH-Accuracy & 8.40 & 2.01 & 10.40 & 0.84 \\
WDH-Accuracy & 9.10 & 1.91 & 9.10 & 1.37 \\
CDH-RT & & & & \\
& 4.05 & 2.82 & 2.23 & 1.02
\end{tabular}


Note. $\mathrm{CDH}=$ Chinese dining hall; $\mathrm{WDH}=$ Western dining hall; $\mathrm{RT}=$ Reaction time; $\mathrm{M}=$ mean; $\mathrm{SD}=$ Standard deviation

\section{DISCUSSION}

The results of our experiment were quite surprising. In contrast to prediction, the results failed to support the hypotheses and illustrated that schematic knowledge hurts a student's memory performance. We assumed that Chinese domestic students would perform better and react faster than international students in Chinese dining hall backgrounds. However, there was no significant difference of accuracy in study abroad experiences or background types, and only a significant difference of accuracy within the interaction has been detected. One possible interpretation is the "false memory effect" of the Deese-Roediger-McDermott (DRM) paradigm [10]. The background of the dining hall produced a common schema of foods, which will then produce memory for words that do not appear in the menu. Take Chinese students as an example, when they see Chinese food names, they will have a higher chance to make a wrong choice. What's more, previous research has proved that schemata cannot support precise retrieval of memory [6]. The more participants depended on their schematic knowledge, the lower their accuracy will be. These data indicate that when participants are familiar with the background, the schema will distort our memories. They will tend to spend more time on guessing schema-based information, rather than the episodic information they remembered just a minute ago and get the wrong answers at last.

Compared to Chinese domestic students, international students have shorter reaction time in both backgrounds, especially in the Chinese background. Considering that most of them have studied abroad for no more than four years, the schema knowledge about Chinese food still has a great influence on them. Based on the former fact, we can speculate that living in a cross-culture environment may reduce the effect of memory distortion caused by schema knowledge. And that might be the reason why international students perform better in both conditions.

\section{LIMITATIONS}

Despite the effective measures and procedures, the study's validity can be affected by some potential threats. These threats to validity can be internal or external. The research might get affected by many confounding variables. They will indirectly influence the results collected from the participants.

Environmental factors. The difference in experimental conditions is an important threat to the internal validity of our research. Since the experiment was held separately by each group member, the environment of this research cannot be uniformly controlled. Time and space of each experiment can be quite different. This can affect the validity of the research because it has been studied that environmental factor may play a large part in research findings that investigators assume are due simply to research designs [11]. In our research, we assume that it is the background picture which causes the schema effect. But the participants can also be influenced by the actual environment unconsciously. Thus, when a participant answers the test questions in different environments, the results can vastly differ from one to another.

Sampling error. A large sampling error can also be a threat to the external validity in this research as a nonprobability sampling method is being used. The participants are mostly our friends and classmates. Individuals were selected based on non-random criteria - only the person we know would be invited to the experiment. This type of sample, known as the convenience sample, is easier and cheaper to access, but has a higher risk of sampling bias. What's more, the sample size of our experiment will also increase sampling error. Since we were working to a very tight deadline, we could only manage to recruit twenty participants, and a sample size of twenty is too small to represent all the international college students and Chinese domestic college students. Considering these potential threats to validity, our future work should therefore focus on improving our sample and condition control. In order to get access to larger sample size, we will do an online survey next time. As for the experiment condition problem, we will ask participants to report the background information before they start the experiment. What's more, we plan to use graphic images instead of texts as stimuli in our next experiment. Different types of stimuli may result in the recalling of different schematic knowledge. By comparing the outcomes of the two experiments, we may have a better understanding of how schemata work.

\section{CONCLUSION}

Throughout history, human beings have never stopped doing research on memory, and we are no exception. The effects of schematic knowledge on memory were investigated experimentally in this paper. Overall, our study revealed that schematic information would hurt individual's memory performance, making them produce non-existent memories. This conclusion could be one possible explanation to memory distortion. This time, we mainly focused on the memory of food menus. In the future, this experimental method could be further used in many other fields, including the memory of stories, names, keywords, and so on. 


\section{REFERENCES}

[1] Alba, J, W., \& Hasher, L. (1983). Is Memory Schematic? Psychological Bulletin, 93(2), 201-231.

[2] Rubínová, E., Blank, H., Koppel, J., \& Ost, J. (2020). Schema and deviation effects in remembering repeated unfamiliar stories. British Journal of Psychology, 112(1), 180-206. https://doi.org/10.1111/bjop.

[3] Bayen, U, J., Nakamura G, V., Dupuis, S, E., \& Yang, C, L. (2000). The use of schematic knowledge in sources in source monitoring. Memory \& Cognition, 28(3), 480-500.

[4] Spainol J., \& Bayen, U, J. (2002). When is Schematic Knowledge Used in Source Monitoring? Journal of Experimental Psychology: Learning, Memory, and Cognition, 28(4), 631-651.

[5] Konopka, A. E., \& Benjamin A. (2009). Schematic Knowledge Changes What Judgements of Learning predict in a source memory task. Memory \& Cognition, 37(1), 42-51.

[6] Brewer, W. F., \& Treyens, J. C. (1981). Role of schemata in memory for places. Cognitive Psychology, 13(2), 207-230. https://doi.org/10.1016/0010-0285(81)90008-6
[7] Hess, T. M., \& Slaughter, S. J. (1990). Schematic knowledge influences on memory for scene information in young and older adults. Developmental Psychology, 26(5), 855-865. https://doi.org/10.1037/0012-1649.26.5.855

[8] Carrell, P. L. (1982, November 30). ERIC EJ291757 - Some Issues in Studying the Role of Schemata, or Background Knowledge, in Second Language Comprehension., Reading in a Foreign Language, $1983 . \quad$ ERIC. https://eric.ed.gov/?id=EJ291757

[9] Qi, H., \& Roberts, K. P. (2021). The influence of culture on children's immediate and delayed retrieval. British Journal of Developmental Psychology, 39, 393-406.

[10] Roediger, H. L., \& McDermott, K. B. (1995). Creating false memories: Remembering words not presented in lists. Journal of Experimental Psychology: Learning, Memory, and Cognition, 21(4), 803-814.

[11] Baldwin, L. (2018). Internal and external validity and threats to validity. In Research concepts for the practitioner of educational leadership (pp. 31-36). Brill Sense. 